\title{
Inteligência Emocional: Validade Convergente e Discriminante do MSCEIT com a BPR-5 e o 16PF
}

Emotional intelligence: Convergent and discriminant validity of the MSCEIT with BPR-5 and 16PF

Ricardo Primi

Universidade São Francisco

José Maurício Haas Bueno

Universidade Presbiteriana Mackenzie e Universidade São

Francisco

Monalisa Muniz

Universidade São Francisco

onn.close(

3CConnection $\overline{7} 7 \mathrm{X}$ idbccor onnCrea = new PooledCo

onnCrea.seth Use (false);

ol.adílement peonnCre

h(Excedtion effor 


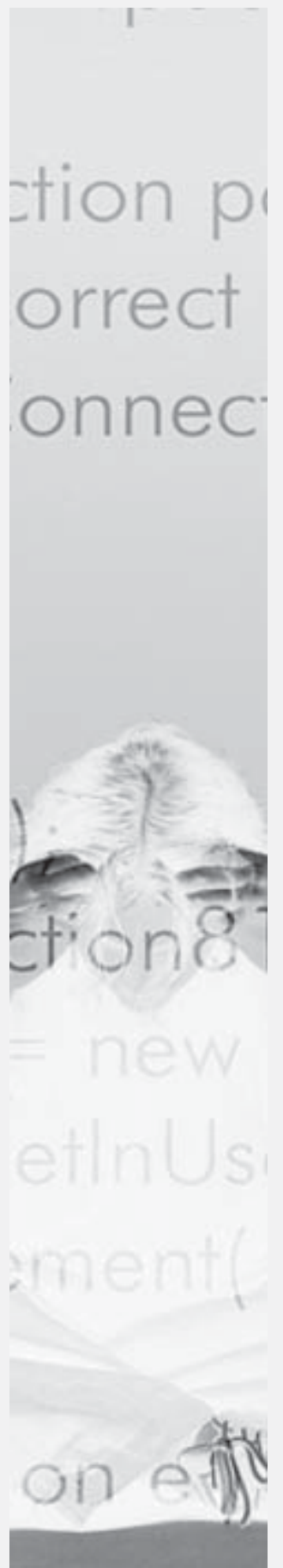

Resumo: A preocupação com a delimitação do construto inteligência emocional foi a principal razão da realização deste trabalho, cujo objetivo foi investigar a validade convergente-discriminante de uma medida de inteligência emocional (MSCEIT), com medidas de personalidade (16PF) e inteligência (BPR-5). A coleta de dados envolveu 107 sujeitos de ambos os sexos, com idades de 17 a 60 anos $(M=29,8 ; D P=9,9)$, funcionários de empresas de diversos segmentos do interior do Estado de São Paulo. Uma análise fatorial exploratória possibilitou a extração de seis fatores ortogonais (rotação varimax) que foram interpretados como relacionados à inteligência, ao neuroticismo, à área estratégica da inteligência emocional, à extroversão, à área experiencial da inteligência emocional e a uma tendência ativo-agressiva. A análise dos dados permitiu concluir que as medidas de inteligência emocional se apresentaram como um construto distinto das medidas tradicionais de inteligência e personalidade, que se referem à capacidade específica de processamento cognitivo com informações emocionais engastadas.

Palavras-chave: avaliação da inteligência, propriedades psicométricas, análise fatorial, emoção, cognição, testes psicológicos.

Abstract: A concern with the delimitation of the emotional intelligence construct was the main reason of the present study, that has investigated the convergent and discriminant validity of a measure of emotional intelligence (MSCEIT) with respect to measures of personality (16PF) and intelligence (BPR-5). Data was collected with 107 participants with ages ranging from 17 to $60(M=29.8 ; S D=9.9)$, employees of various enterprises located outside São Paulo State downtown area. An exploratory factor analysis has led to the extraction of six orthogonal factors (varimax rotation) that were interpreted as: intelligence, neuroticism, strategic area of emotional intelligence, extroversion, experiential area of emotional intelligence and an active-aggressive orientation. Data analysis has led to the conclusion that emotional intelligence measures turned out to be distinct of traditional measures of intelligence and personality, referring to the specific capacity of cognitive processing embedded with emotional information.

Key words: intelligence assessment, psychometric properties, factor analysis, emotion, cognition, psychological testing. 
O enfoque cognitivista éo mais recente, e centra sua atenção na natureza das tarefas e nos processos cognitivos internos usados para sua resolução. lém da exploração da mão-de-obra infanto-juvenil nos países pobrese em desenvo/vimento, empaíses desenvolvidos, as crianças também são maltratadas.
O estudo da inteligência tem-se constituído em uma das mais fecundas áreas da Psicologia nos últimos 100 anos. Primi (2003) encontrou mais de 18400 artigos com a palavra inteligência em seu título, em uma rápida pesquisa na base de dados PsycINFO da Associação Americana de Psicologia. Esses estudos, de acordo com a concepção metodológica e conceitual que empregam, podem ser classificados em três categorias: a psicométrica, a desenvolvimentalista e a cognitivista (Almeida, 1988).

O enfoque desenvolvimental tem Jean Piaget como principal expoente. Sua concepção se aproxima de uma visão biológica evolutiva dos seres vivos, em que a inteligência é vista como o elemento principal que promove a adaptação do indivíduo ao meio. Seu desenvolvimento segue um curso comum para todas as pessoas. O enfoque cognitivista é o mais recente, e centra sua atenção na natureza das tarefas e nos processos cognitivos internos usados para sua resolução. A proximidade de seus conceitos com elementos da computação resultou na denominação de teoria do processamento da informação. A perspectiva psicométrica da inteligência, adotada neste trabalho, realiza estudos sobre capacidades com base em análises estatísticas, aplicadas sobre as pontuações de sujeitos em diferentes tipos de tarefa. Basicamente, são investigadas as relações dos diferentes tipos de tarefa entre si, e dessas com outras mensurações, como personalidade, desempenho acadêmico, profissional, etc. $\mathrm{O}$ principal recurso estatístico empregado nesses estudos é uma análise multivariada de matrizes de correlações chamada análise fatorial.

A principal concepção da análise fatorial é a de que um conjunto aparentemente complexo de medidas de variáveis observáveis pode ser simplificado por meio de um conjunto menor de variáveis hipotéticas nãoobserváveis, as variáveis-fonte (Pasquali,
2003). Estas últimas seriam a causa subjacente que produziria as associações entre as variáveis observadas que aparecem agrupadas na matriz de correlação.

Esse princípio, aplicado ao estudo da inteligência, ajuda a descobrir quais os tipos de tarefas que podem ser agrupadas em um mesmo fator, e, porque o agrupamento é devido a um fator comum, por meio da análise das demandas cognitivas dessas tarefas, seria possível definir o tipo de processamento intelectual comum às tarefas. Assim, por exemplo, quando as provas de fluência verbal, vocabulário, associação de palavras se agrupam em um mesmo fator, supõe-se que, subjacente a elas, esteja algum tipo de raciocínio comum a todas, denominado raciocínio verbal.

Dessa forma, no contexto da proposição de uma nova inteligência, esse procedimento também pode ser aplicado investigando como as eventuais tarefas que se constituem indicadores da nova capacidade se relacionam com outros tipos de inteligência, cuja existência já tenha sido solidamente comprovada por pesquisas anteriores. Nesses casos, espera-se que haja alguma correlação entre a nova e outros tipos de inteligência já estabelecidos na literatura com magnitude suficiente para ser considerada uma inteligência, mas deve preservar alguma variância única para ser considerada diferente das anteriores.

A inteligência emocional é um tipo de inteligência proposto recentemente (Salovey \& Mayer, 1990) que se refere às capacidades mentais associadas ao processamento de informações e que contenham afetos imbricados. Em razão do conteúdo específico de informação envolvida, os autores propuseram a existência de uma especialização do cérebro para lidar com elas. Quatro habilidades estariam relacionadas à capacidade geral de processamento dessas informações: a percepção de emoções, a utilização das 
emoções como facilitadoras do pensamento, o conhecimento emocional e a regulação das emoções (Mayer \& Salovey, 1997).

A percepção das emoções abrange desde a capacidade de identificar emoções em si mesmo, em outras pessoas e em objetos ou condições físicas, até a capacidade de expressar essas emoções e as necessidades a elas relacionadas, e ainda a capacidade de avaliar a autenticidade de uma expressão emocional, detectando sua veracidade, falsidade ou tentativa de manipulação.

A emoção, como facilitadora do ato de pensar, diz respeito à influência que as emoções têm nos processos cognitivos, e, ao mesmo tempo, à eficácia com que a pessoa compreende e utiliza a informação desse sistema de alerta que dirige a atenção e o pensamento para as informações (internas ou externas) mais importantes no processo de solução de problemas. A capacidade de gerar sentimentos em si mesmo, por exemplo, pode ajudar uma pessoa em situações de tomada de decisão, funcionando como um "ensaio", no qual as emoções podem ser geradas, sentidas, manipuladas e examinadas antes de se decidir. O conhecimento emocional inclui desde a capacidade de nomear as emoções, englobando a capacidade de identificar e nomear diferenças e nuances entre elas (como gostar e amar), até a compreensão de sentimentos complexos, como amar e odiar uma mesma pessoa, bem como as transições de um sentimento para outro, como a de raiva para a vergonha, por exemplo.

Finalmente, o gerenciamento reflexivo das emoções refere-se às estratégias efetivas de gerência das experiências emocionais para poder alterá-las, em si e nos outros, de forma a produzir efeitos desejados e condizentes com a solução de problemas. Essa capacidade diz respeito à tolerância e compreensão na medida certa das reações emocionais, agradáveis ou desagradáveis, sem exagero ou diminuição de sua importância, no controle ou expressão no momento apropriado para promover o crescimento emocional e intelectual.

Estudos correlacionais e analítico-fatoriais anteriores têm chegado a resultados diferentes dependendo do procedimento escolhido para mensurá-la. Obtém-se um tipo de resultado quando os instrumentos são de auto-relato, os quais são diferentes dos resultados obtidos quando são empregados instrumentos de desempenho máximo. Os principais instrumentos por auto-relato empregados até o momento foram o Bar-On Emotional Quocient Inventory - EQ-i (BAR-ON, 1996, 1997) e o Emotional Intelligence Scale (Schutte et al., 1998), enquanto os principais instrumentos por desempenho foram a Multifactor Emotional Intelligence Scale - MEIS (Mayer, Salovey \& Caruso, 1997) e sua versão aprimorada, o Mayer-Salovey-Caruso Emotional Intelligence Test - MSCEIT (Mayer, Salovey \& Caruso, 2002).

Em geral, os estudos têm demonstrado que, quando avaliada por auto-relato, a inteligência emocional apresenta um fator geral e, dependendo do instrumento, de 3 a 6 subescalas hierarquicamente subordinadas ao fator geral. Ao lado disso, tem apresentado fortes correlações com traços de personalidade, principalmente com os cinco grandes fatores (CGF), e baixas correlações com medidas de desempenho. Esses dados atestam que o que é avaliado por esses inventários não atende aos critérios que devem ser preenchidos para se definir uma inteligência, mas, sim, como uma definição de aspectos da personalidade associados à adaptação. A seguir, os principais estudos dessa linha serão revisados.

O Emotional Intelligence Scale (Schutte et al., 1998), por exemplo, é uma medida de inteligência emocional baseada no modelo de Salovey e Mayer (1990). No desenvolvimento do instrumento, a análise fatorial aplicada às respostas de 346 adultos resultou em uma
A emoção, como facilitadora do ato de pensar, diz respeito à influência que as emoções têm nos processos cognitivos, e, ao mesmo tempo, à eficácia com que a pessoa compreendee utiliza a informação desse sistema de alerta que dirige a atenção e o pensamento para as informações (internas ou externas) mais importantes no processo de solução de problemas. 
escala unifatorial com 33 itens, com bons índices de consistência interna e de precisão por teste-reteste. Essa escala apresentou correlações positivas e significativas com outras medidas por auto-relato, como alexitimia, atenção às emoções, clareza quanto às emoções, reparação do humor, otimismo, controle dos impulsos e com o traço de abertura à experiência do modelo dos cinco grandes fatores (CGF). Ao lado disso, apresentou validade preditiva para notas escolares, mas não se correlacionou com testes de habilidade cognitiva. Resultados semelhantes foram obtidos com a versão espanhola dessa escala, estudada em adultos e adolescentes normais (Chico, 1999). Os escores em inteligência emocional apareceram associados ao traço de personalidade extroversão, mas não ao QI nem ao desempenho acadêmico.

Outro estudo com esse instrumento, aplicado em adolescentes superdotados, mostrou resultados diferentes, especialmente em relação à unifatorialidade (Chan, 2003). O autor encontrou quatro fatores: habilidades sociais, auto-regulação das emoções, empatia e utilização das emoções. Austin et al. (2004) acrescentaram e inverteram itens da Emotional Intelligence Scale, mas esse procedimento acabou por não melhorar as propriedades psicométricas da escala. Ao lado disso, e em oposição a estudos anteriores, apenas três fatores foram identificados para a nova escala de 41 itens: otimismo/regulação do humor, utilização das emoções e avaliação das emoções.

Fatores semelhantes foram encontrados por Tapia (2001) em um trabalho em que desenvolveu uma escala com 41 itens e boas propriedades psicométricas para a avaliação do traço de inteligência emocional. Uma análise fatorial pela máxima verossimilhança e rotação varimax revelou a existência de quatro fatores: empatia, utilização dos sentimentos, habilidade em relacionamentos e autocontrole.
Confusão semelhante em relação à estrutura fatorial ocorre entre as pesquisas realizadas com o Bar-On Emotional Quotient Inventory - EQ-i (BAR-ON, 1997). Originalmente, esse instrumento é constituído por 133 itens, que se agrupam formando cinco escalas e quinze subescalas: escalas intrapessoais (auto-respeito, autoconsciência emocional, assertividade, independência e auto-atualização), escalas interpessoais (empatia, responsabilidade social e relacionamento interpessoal), escalas de adaptabilidade (teste de realidade, flexibilidade e solução de problemas), escalas de gerenciamento do stress (tolerância ao stress, controle dos impulsos) e escalas dos humores gerais (otimismo e felicidade). No entanto, outros estudos com o instrumento questionam essa estrutura fatorial. Por exemplo, Sutarso (1999) também encontrou uma solução com cinco fatores, mas definidos de maneira diferente (automotivação, empatia, habilidade para relacionamentos, autoconhecimento e gerenciamento emocional), e Palmer et al. (2003), por meio de análises fatoriais exploratórias e confirmatórias, forneceram evidências para um fator geral de inteligência emocional e seis fatores primários.

Uma análise fatorial aplicada por Kohan (2003) sobre os resultados do Emotional Quocient Inventory (EQ-i; BAR-ON, 1997) e o Emotional Intelligence Scale (EIS) (Shutte et al., 1998) e em um instrumento de avaliação da personalidade baseado no modelo dos cinco grandes fatores revelou que não há independência entre as medidas de inteligência emocional por auto-relato e o domínio dos cinco grandes fatores, exceto para dois dos fatores teoricamente relacionados à IE: avaliação e utilização das emoções.

Petrides e Furnham (2000, 2001) e Kohan (2003) encontraram relações entre ambos, o Bar-On Emotional Quotient Inventory e o Emotional Intelligence Scale e os traços do modelo dos cinco grandes fatores e o de Eysenck. Um outro instrumento de auto-relato 
construído por Saklofske, Austin e Minski (2003) foi submetido a análises fatoriais exploratória e confirmatória, que revelaram um fator hierarquicamente superior relacionado à inteligência emocional, e a quatro fatores a ele subordinados. O fator geral correlacionouse negativamente com neuroticismo e positivamente com extroversão, abertura, socialização (agreeableness) e responsabilidade (conscientiousness).

Em resumo, os estudos sobre a inteligência emocional avaliada por questionários de autorelato têm demonstrado que as medidas não se diferenciam muito dos traços de personalidade já conhecidos, podendo ser explicadas, em grande parte, por eles. Por outro lado, quando os estudos empregam instrumentos de inteligência emocional baseados em desempenho, isto é, instrumentos que apresentam tarefas cuja resolução depende da utilização da função cognitiva que se quer avaliar, os estudos têm demonstrado que as variáveis medidas se correlacionam mais com outras medidas de desempenho (outros tipos de raciocínio) e menos com medidas de traços de personalidade.

Mayer, Salovey e Caruso (2000), por exemplo, realizaram um estudo bastante completo sobre os requisitos que uma "inteligência" deve preencher para ser legitimada como tal. Análises estatísticas sobre as pontuações na MEIS, entre outros resultados, revelaram que a inteligência emocional: correlacionou-se moderadamente com medidas de inteligência verbal, indicando que possui alguma variância em comum com outra forma de inteligência, mas também alguma variância única; mostrouse promissora como preditora de outras qualidades, tais como empatia, estilo parental (calorosidade emocional dos pais, capacidade para ouvir os filhos, conduta não-abusiva, flexibilidade, etc.) e atividades de vida nos aspectos culturais, crescimento pessoal e entretenimento; produziu quatro fatores, sendo um fator geral de inteligência emocional que pode ser subdividido nas subescalas de percepção, compreensão e controle emocional, reduzindo o modelo inicial de quatro níveis para um modelo com três níveis.

Um outro grupo de pesquisadores, no entanto, encontrou resultados menos encorajadores. Davies, Stankov e Roberts (1998) publicaram um artigo contendo três estudos, cujos principais objetivos eram: (a) examinar o status empírico das medidas de inteligência emocional por autorelato e por desempenho em tarefas específicas; (b) determinar as relações que a inteligência emocional possa ter com habilidades cognitivas tradicionais, incluindo índices de inteligência fluida, cristalizada e social, e (c) examinar a relação entre os vários tipos de medidas de inteligência emocional e variáveis de personalidade, incluindo medidas dos seis traços de personalidade (extroversão, neuroticismo, psicoticismo, conscienciosidade, socialização e abertura).

O primeiro estudo incluiu uma ampla gama de medidas de inteligência emocional, tanto por auto-relato quanto por desempenho, além de outras, como habilidades cognitivas, inteligência fluida e cristalizada, inteligência social e traços de personalidade. As principais medidas de inteligência emocional por desempenho eram as provas de percepção de emoções em faces, cores, músicas e sons, com pequenas modificações em relação às utilizadas por Mayer, DiPaolo e Salovey (1990).

Foram encontradas algumas evidências que apóiam e outras que não apóiam a validade das medidas e do construto. Por exemplo, a favor da validade, contam as correlações negativas encontradas entre medidas de alexitimia e de inteligência emocional (avaliada tanto por autorelato quanto por desempenho) e as intercorrelações entre as quatro escalas de percepção de emoções (faces, cores, músicas e sons), que confirmam a hipótese de Mayer e DiPaolo e Salovey (1990) de que o julgamento consensual de emoções se generaliza por entre diferentes tipos de estímulos. 
Contra essas evidências, contudo, conta o fato de que essas mesmas quatro escalas, apesar de se apresentarem agrupadas isoladamente em um único fator (percepção de emoções), não se correlacionaram significativamente nem com medidas de inteligência emocional por auto-relato nem com variáveis de habilidades cognitivas e personalidade. Isso contradiz o postulado de Salovey e Mayer de que tais habilidades constituiriam uma capacidade cognitiva conceitualmente relacionada à inteligência. Ao lado disso, altas correlações entre medidas de inteligência emocional por auto-relato e traços de personalidade, como também os resultados que os estudos anteriormente citados encontraram, levaram os autores a sugerir que a inteligência emocional pode não ser inteiramente distinta de construtos relacionados à personalidade. Uma análise fatorial exploratória com rotação oblíqua realizada sobre as trinta variáveis incluídas nesse primeiro estudo resultou em oito fatores. O primeiro deles foi capaz de explicar apenas 15\% da variância total, considerado muito fraco para confirmar a validade unifatorial do construto estudado. Os oito fatores encontrados foram os seguintes: neurose, psicose, extroversão, clareza emocional, conhecimento emocional, percepção de emoções, inteligência cristalizada e inteligência fluida. Esse conjunto de fatores foi capaz de explicar $64 \%$ da variância total encontrada.

Entre esses fatores, aqueles que supostamente estariam relacionados à inteligência emocional, conforme proposta por Salovey e Mayer (1990), clareza emocional, conhecimento emocional e percepção de emoções (faces, cores, música e sons), se mostraram independentes tanto da inteligência cristalizada quanto da inteligência fluida. Além disso, os autores também constataram que outros componentes da inteligência emocional, como regulação emocional, expressão emocional, impulsividade e empatia, apresentaram cargas em fatores tipicamente relacionados a dimensões da personalidade, como neuroticismo, extroversão e psicoticismo, cujos construtos já foram extensivamente demonstrados como independentes de habilidades cognitivas.

Em outro estudo, Roberts, Zeidner e Matthews (2001) aplicaram a MultiFactor Emotional Intelligence Scale (MEIS), o Trait Self-Description Inventory (TSDI), uma medida dos cinco grandes fatores de personalidade, e a Armed Services Vocational Aptitude Battery (ASVAB, uma medida de inteligência) em 704 estudantes universitários. Nesse estudo, os autores consideram que instrumentos de avaliação por desempenho são mais apropriados do que os de auto-relato para mensuração da inteligência emocional como um construto distinto da personalidade, mas os resultados, em geral, foram duvidosos. Embora a MEIS tenha mostrado validade divergente (correlação mínima com o TSDI), sistemas de pontuações diferentes (por concordância com o consenso e com especialistas) levaram a resultados contraditórios. A análise da estrutura fatorial e da fidedignidade das escalas identificou mais problemas de mensuração. Na maioria das vezes, foi questionável se a MEIS representa uma operacionalização válida e fidedigna da inteligência emocional.

Como a MEIS foi o primeiro instrumento por desempenho criado pelos proponentes da inteligência emocional para avaliação das quatro habilidades a ela relacionadas, atualmente, eles argumentam que o MSCEIT, versão mais atual do MEIS, superou os principais problemas observados pelos pesquisadores. Em um dos estudos com o MSCEIT para verificação do ajustamento entre as soluções com um, dois e quatro fatores e o modelo teórico de inteligência emocional, Mayer et al. (2003) realizaram uma análise fatorial confirmatória, que apresentou ajustamento progressivo ao modelo conforme o aumento do número de fatores, embora todos tenham apresentado bons índices de 
ajustamento. Esses dados levaram à conclusão que a análise fatorial confirmatória apóia o modelo teórico proposto com um, dois e quatro fatores. Os autores também argumentam que o principal problema apontado por Roberts et al. (2001) com o MEIS, sobre a inconsistência entre os sistemas de pontuação, foi resolvido no MSCEIT, pois, nele, as correlações entre as pontuações por um ou outro método atingem valores ao redor de 0,90 .

Em outro estudo, Bachard (2003) submeteu os resultados de 31 medidas relacionadas à inteligência emocional e outras relacionadas à personalidade e inteligência a análises fatoriais e correlacionais. Os resultados indicaram que muitas das medidas de inteligência emocional e social por auto-relato coincidem com dimensões de personalidade, e não com habilidades cognitivas. Contudo, a habilidade de perceber emoções em outras pessoas, em objetos inanimados e em outras sensações surgiu como uma habilidade cognitiva única. Medidas desse construto formaram consistentemente um fator único, que, por sua vez, obteve uma carga fatorial considerável em um fator de ordem superior identificado como inteligência cristalizada. Um dos problemas levantados é que essas medidas podem ser associadas à habilidade verbal, sendo necessária a realização de pesquisas adicionais para determinar se elas são ou não novas medidas relacionadas à habilidade verbal. Brackett e Mayer (2003) realizaram estudos de validade convergente, discriminante e incremental do Emotional Intelligence Scale, o Bar-On EQ-i e o MSCEIT. Verificaram que o MSCEIT (medida por desempenho) apresenta características distintas dos outros dois instrumentos (medidas por auto-relato), na medida em que a correlação entre o MSCEIT e as medidas por auto-relato, apesar de significativas, foram muito baixas (0,18 e 0,21, respectivamente) em relação à correlação entre as medidas por auto-relato entre si $(0,43)$. Ao lado disso, aplicaram uma análise fatorial sobre as pontuações em inteligência emocional pelos três instrumentos, medidas dos cinco grandes fatores de personalidade, medidas de bem-estar psicológico e uma medida cognitiva (SAT verbal), e encontraram como resultado os três fatores caracterizados por cada uma das medidas de inteligência emocional. Constataram que o MSCEIT apresentou validade discriminante das medidas de personalidade e bem-estar psicológico, enquanto o EQ-i e o Emotional Intelligence Scale apresentaram considerável variância comum com essas medidas, respectivamente. Verificaram, ainda, que a convergência do MSCEIT se deu com a medida de desempenho cognitivo (SAT verbal), que apresentou carga no mesmo fator que as subescalas do MSCEIT. Nesse mesmo estudo, os autores também investigaram a validade incremental dos três instrumentos em relação a critérios externos (uso de drogas, uso de álcool, fumo, desvio social, ranking do desempenho escolar e média escolar) quando controlados por variáveis relacionadas à personalidade (cinco grandes fatores) e ao desempenho cognitivo (SAT verbal). O resultado mostrou que as pontuações no MSCEIT continuavam capazes de predizer desvio social, e as pontuações do EQ-i continuavam prevendo uso de álcool. As pontuações no Emotional Intelligence Scale apresentaram correlação negativa com desempenho acadêmico.

No Brasil, alguns estudos já foram feitos no Laboratório de Avaliação Psicológica e Educacional (LabAPE) da Universidade São Francisco, com versões traduzidas para o português, dos testes de desempenho. Por exemplo, Bueno e Primi (2003) aplicaram a prova de percepção de emoções da MEIS em estudantes de Psicologia, e encontraram correlação positiva com a escala de administração da imagem e negativa com o traço de imaginação do 16PF. Também foi encontrada correlação positiva entre percepção de emoções e raciocínio espacial.

Dantas (2004) fez uma análise fatorial com rotação oblíqua das pontuações no MSCEIT e 
encontrou dois fatores, os quais corresponderam às áreas estratégica e experiencial da inteligência emocional. Em outro estudo, empregando rotação ortogonal, Jesus Jr. (2004) encontrou uma estrutura com três fatores, sendo que o primeiro fator foi relacionado à área experiencial e os outros dois restantes subdividiram a área estratégica. No trabalho de Cobêro (2004), a análise fatorial dos subtestes do MSCEIT também extraiu dois fatores, um relacionado à área estratégia e o outro, à área experiencial, como em Dantas (2004). No entanto, o primeiro fator continha um subteste que teoricamente deveria estar agrupado no segundo fator. Esses resultados mostram que a versão em português é relativamente coerente com o modelo bifatorial proposto pelos autores.

Ainda não há evidências seguras de que a inteligência emocional, operacionalizada pelo MSCEIT, se constitua uma capacidade cognitiva especifica.
No entanto, como se verificou na literatura, ainda são ambíguos os dados que evidenciam se tais fatores presentes no MSCEIT, destinados a operacionalizar a inteligência emocional, se configuram como capacidades cognitivas separadas das já conhecidas e independentes das medidas de personalidade. Ainda não há evidências seguras de que a inteligência emocional, operacionalizada pelo MSCEIT, se constitua uma capacidade cognitiva específica. Parece ser mais seguro que os subtestes do MSCEIT divirjam em medidas de traços de personalidade, mas ainda não são tão claras as associações com medidas cognitivas.

Aqui se apresenta um estudo fatorial das correlações entre o MSCEIT, um teste de inteligência fluida, inteligência cristalizada e processamento visual e um teste de personalidade, buscando verificar a validade convergente e discriminante do MSCEIT em sua versão brasileira. Espera-se oferecer mais dados para a discussão sobre a definição do construto inteligência emocional como uma capacidade específica. Com base na literatura, espera-se que o MSCEIT apresente-se convergente com medidas de inteligência e divergente das medidas de traços de personalidade. Espera-se que a magnitude da convergência seja tal (nem muito alta nem muito baixa) que sustente a existência dos fatores como capacidades distintas relacionadas à inteligência, mas distinta das formas já conhecidas. Também se espera que a estrutura fatorial seja condizente com o modelo teórico de dois fatores, como vem sendo encontrado. Esses dados fazem parte de um projeto mais amplo que busca investigar as propriedades psicométricas do MSCEIT traduzido para a língua portuguesa.

\section{Método}

\section{Participantes}

A coleta de dados foi realizada, por conveniência dos pesquisadores, com 107 sujeitos, funcionários de empresas de diversos segmentos, situadas em Municípios do interior do Estado de São Paulo. As idades relatadas variaram de 17 a 60 anos, com média igual a 29,8 e desvio padrão igual a 9,9. Quanto ao gênero, $65,7 \%$ da amostra ficou constituída por homens, $34,3 \%$, por mulheres (1,9\% não informaram o sexo). A distribuição de freqüências também indicou que 49,5\% da amostra foi constituída por pessoas solteiras, $47,6 \%$, por pessoas casadas, $1,9 \%$, por desquitados/divorciados e 1,0\%, viúvos (3,7\% não informaram o estado civil). Quanto à escolaridade, a amostra ficou constituída por $2,0 \%$ de pessoas com ensino fundamental completo ou incompleto; $67,3 \%$, de pessoas com ensino médio completo ou incompleto, normal ou técnico; $30,8 \%$, de pessoas com nível superior completo ou incompleto $(2,8 \%$ não informaram escolaridade).

\section{Instrumento}

\section{Mayer-Salovey-Caruso Emotional Intelligence Test (MSCEIT)}

Esse instrumento é composto por 141 itens, distribuídos em 8 seções, conforme mostra a 
Tabela 1. As seções A (faces) e E (figuras) são destinadas à avaliação da capacidade de perceber emoções em faces e paisagens, respectivamente; as seções B (facilitação) e F (sensação) são compostas por tarefas relacionadas à utilização da emoção para facilitação do pensamento; a compreensão de emoções é avaliada pelas tarefas propostas nas seções C (transição) e G (mistura), e, finalmente, o gerenciamento das emoções é avaliada por meio das tarefas das seções D (administração de emoções) e H (relações emocionais). Na Tabela 1, também estão demonstrados os diferentes níveis de combinação dos subtestes para se obterem escores para quatro facetas, duas áreas e um escore geral. Neste estudo, utilizamos os escores nos subtestes.O método adotado para pontuação foi o da atribuição de pontos proporcionais à concordância com o consenso. Segundo esse critério, o sujeito recebe pontos proporcionalmente ao número de pessoas que escolheram a mesma alternativa que ele. Assim, se o sujeito escolheu uma alternativa com $80 \%$ da amostra, então sua pontuação, naquele item, é de 0,80 . Os sujeitos obtiveram um escore em cada tarefa: faces, figuras, facilitação, sensações, transição, misturas, administração das emoções e relações emocionais.

Tabela 1. Organização dos sub-testes do MSCEIT

\begin{tabular}{|c|c|c|c|c|}
\hline Escala geral & Área & Faceta & Subteste & Seção \\
\hline \multirow{8}{*}{$\begin{array}{l}\text { Emocional } \\
\text { Inteligência }\end{array}$} & \multirow{4}{*}{$\begin{array}{c}\text { Experiencial } \\
\text { (IEE) }\end{array}$} & Percepção das emoções & Faces & A \\
\hline & & & Figuras & $E$ \\
\hline & & Facilitação do pensamento & Facilitação & B \\
\hline & & & Sensações & $\mathrm{F}$ \\
\hline & \multirow{4}{*}{$\begin{array}{l}\text { Estratégica } \\
\text { (IES) }\end{array}$} & Compreensão das emoções & Transições & $\mathrm{C}$ \\
\hline & & & Misturas & G \\
\hline & & Gerenciamento das emoções & $\begin{array}{l}\text { Administração de } \\
\text { emoções }\end{array}$ & D \\
\hline & & & Relações emocionais & $\mathrm{H}$ \\
\hline
\end{tabular}

Inventário dos 16 fatores de personalidade (16PF).

Esse instrumento investiga traços de personalidade por meio de um inventário constituído por 185 itens. Foram obtidas as pontuações em duas escalas de controle (administração da imagem - Al e aquiescência - AQ) e nos dezesseis fatores primários denominados por letras: A - Expansividade, B - Inteligência, C - Estabilidade emocional, E - Afirmação, F - Preocupação, G Consciência, H - Desenvoltura, I-Brandura, L - Confiança, M - Imaginação, N - Requinte, O - Apreensão, Q1 - Abertura a Novas Experiências, Q2 - Auto-suficiência, Q3 Disciplina, Q4 - Tensão (Cattell, Cattell \& Cattell, 1993).
Bateria de Provas de Raciocínio (BPR-5)

A Bateria de Provas de Raciocínio inclui cinco provas: raciocínio verbal $(\mathrm{RV})$, raciocínio abstrato (RA), raciocínio mecânico (RM), raciocínio espacial (RE) e raciocínio numérico (RN). A prova de raciocínio verbal avalia a extensão de vocabulário e a capacidade de estabelecer relações abstratas entre conceitos verbais; a prova de raciocínio abstrato avalia a capacidade de estabelecer relações abstratas em situações novas para as quais se possui pouco conhecimento previamente aprendido; a prova de raciocínio mecânico avalia o conhecimento prático de mecânica e física e a capacidade de integrar as informações em textos com a figura descritiva da situação- 
problema; a prova de raciocínio espacial avalia a capacidade de formar representações mentais visuais e manipulá-las, transformando-as em novas representações; por fim, a prova de raciocínio numérico avalia a capacidade de raciocinar indutiva e dedutivamente com símbolos numéricos em problemas de indução e o conhecimento e a facilidade de executar as operações aritméticas básicas.

As provas de raciocínio verbal, abstrato e mecânico são compostas por 25 itens e são aplicadas com limite de tempo de 10, 12 e 15 minutos, respectivamente. As provas de raciocínio espacial e numérico são compostas por 20 itens, e são aplicadas em, no máximo, 18 minutos cada uma. Para cada resposta certa, foi atribuído um ponto, e cada sujeito obteve uma pontuação em cada um dos cinco tipos de raciocínio avaliados.

Procedimentos

Os instrumentos foram aplicados da forma coletiva, utilizando-se dois dias para essa coleta de dados, que ocorreu nas empresas, em salas cedidas pelas mesmas. No primeiro dia, foi aplicado o 16PF e a primeira parte da BPR-5, e, no segundo dia, aplicou-se o MSCEIT e a segunda parte da BPR-5.

Resultados e Discussão

Os resultados são apresentados em dois blocos: o primeiro apresenta as estatísticas descritivas dos instrumentos utilizados e o segundo apresenta os resultados da análise fatorial. A Tabela 2 apresenta as estatísticas descritivas das escalas do 16PF. Esse instrumento utiliza o resultado Padrão Dez (Sten), cuja média é igual a 5,5, e o desvio padrão igual a 2. Os resultados dos sujeitos foram previamente convertidos para essa escala padronizada (com exceção das escalas Al e AQ, que são expressas em percentis).

Tabela 2. Estatísticas descritivas do 16PF

\begin{tabular}{|c|c|c|c|c|c|}
\hline & $\mathrm{N}$ & Mínimo & Máximo & Média & $\begin{array}{l}\text { Desvio } \\
\text { Padrão }\end{array}$ \\
\hline Administração da imagem (Al) & 107 & 6 & 99 & 72,64 & 23,606 \\
\hline Aquiescência (AQ) & 107 & 1 & 97 & 36,00 & 26,799 \\
\hline Expansividade (A) & 107 & 1 & 9 & 4,41 & 2,028 \\
\hline Inteligência (B) & 107 & 3 & 10 & 7,09 & 1,926 \\
\hline Estabilidade emocional (C) & 107 & 1 & 9 & 6,42 & 1,894 \\
\hline Afirmação (E) & 107 & 1 & 8 & 5,36 & 1,834 \\
\hline Preocupação (F) & 107 & 1 & 9 & 4,41 & 1,764 \\
\hline Consciência (G) & 107 & 3 & 10 & 7,29 & 1,791 \\
\hline Desenvoltura $(\mathrm{H})$ & 107 & 2 & 9 & 5,97 & 1,840 \\
\hline Brandura (I) & 107 & 1 & 10 & 4,36 & 1,919 \\
\hline Confiança (L) & 107 & 1 & 9 & 4,45 & 2,071 \\
\hline Imaginação (M) & 107 & 2 & 9 & 4,07 & 1,515 \\
\hline Requinte (N) & 107 & 1 & 9 & 5,56 & 1,889 \\
\hline Apreensão (O) & 107 & 1 & 10 & 4,93 & 1,870 \\
\hline Abertura a mudanças (Q1) & 107 & 1 & 10 & 5,57 & 1,943 \\
\hline Auto-suficiência (Q2) & 107 & 2 & 10 & 4,63 & 2,049 \\
\hline Disciplina (Q3) & 107 & 2 & 9 & 6,60 & 1,868 \\
\hline Tensão (Q4) & 107 & 1 & 8 & 4,29 & 1,764 \\
\hline
\end{tabular}

As médias dos participantes obtidas nos fatores do 16PF indicam que esse grupo é similar ao grupo 
de padronização e que as pontuações dos sujeitos se distribuíram bem ao longo da amplitude máxima possível e com boa variabilidade em todas as escalas. Foram encontrados resultados ligeiramente acima do esperado nos fatores B e G e abaixo do esperado em M.

As estatísticas descritivas das provas de raciocínio são apresentadas na Tabela 3. Aqui também as notas foram convertidas em resultados padronizados com Média $=100$ e Desvio Padrão $=15$, com auxílio das tabelas do manual.

Tabela 3- Estatísticas descritivas das provas de raciocínio da BPR-5.

\begin{tabular}{|l|l|l|l|l|l|}
\hline & N & Mínimo & Máximo & Média & $\begin{array}{r}\text { Desvio } \\
\text { padrão }\end{array}$ \\
\hline Raciocínio abstrato - EPN & 107 & 60 & 131 & 89,92 & 14,255 \\
\hline Raciocínio verbal - EPN & 107 & 65 & 132 & 90,36 & 13,166 \\
\hline Raciocínio mecânico - EPN & 107 & 70 & 141 & 96,54 & 14,175 \\
\hline Raciocínio espacial - EPN & 107 & 60 & 124 & 87,03 & 13,359 \\
\hline Raciocínio numérico - EPN & 107 & 70 & 146 & 91,54 & 13,499 \\
\hline
\end{tabular}

As pontuações dos sujeitos nas provas de raciocínio também apresentaram boa distribuição, ocupando uma boa extensão da amplitude máxima possível e com boa variabilidade em torno da média. Pode-se observar que, na BPR-5, os sujeitos apresentam desempenho ligeiramente abaixo das expectativas normativas. A Tabela 4 apresenta as estatísticas descritivas das provas de inteligência emocional.

Tabela 4-Estatísticas descritivas das provas de inteligência emocional

\begin{tabular}{|c|c|c|c|c|c|}
\hline Sub-testes do MSCEIT & $\mathrm{N}$ & Mínimo & Máximo & Média & Desvio \\
\hline & & & & & padrão \\
\hline Faces & 107 & 15,09 & 51,73 & 40,41 & 7,488 \\
\hline Figuras & 107 & 10,19 & 54,36 & 42,87 & 8,760 \\
\hline Facilitação & 107 & 17,70 & 52,63 & 42,05 & 7,772 \\
\hline Sensações & 107 & 11,23 & 53,04 & 37,70 & 9,289 \\
\hline Transições & 107 & 22,54 & 54,10 & 42,91 & 5,773 \\
\hline Misturas & 107 & 19,48 & 50,40 & 36,91 & 7,006 \\
\hline Administração de emoções & 107 & 12,27 & 56,85 & 41,44 & 8,694 \\
\hline Relações emocionais & 107 & 11,00 & 55,23 & 39,18 & 10,345 \\
\hline
\end{tabular}

Nota-se que, com exceção das provas de sensação e mistura, a distribuição de pontuações se deu ao redor de uma média situada mais próxima ao topo da amplitude máxima obtida, mas sempre com boa variabilidade. As boas variabilidades obtidas nas aplicações resultaram em uma matriz de correlações razoavelmente adequada para a realização da análise fatorial (ver Tabela 5), com a medida de adequação da amostra de Kaiser-Meyer-Olkin igual a 0,647 e o teste de esfericidade de Bartlett, indicando que a matriz de correlações não é uma matriz identidade $(\div 2=1312,6, g l=496$, sig. $=0,000)$.
Inicialmente, a aplicação de uma análise fatorial por componentes principais e rotação oblimin mostrou que as correlações entre os fatores eram, em sua maioria, próximas de zero, sendo que a correlação de maior magnitude não passou de 0,20 . Sendo assim, optou-se pela aplicação da rotação varimax, que se adequou aos dados e é mais parcimoniosa. A primeira solução revelou a existência de dez fatores com eigenvalues superiores a um, explicando $68,8 \%$ da variância total. Contudo, a análise do scree-plot (Figura 1) revelou a existência de seis fatores mais significativos 


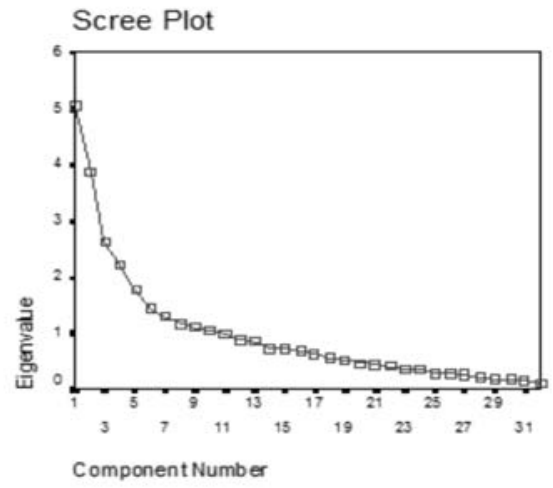

Com base na análise do scree-plot, optou-se pela aplicação de uma nova análise fatorial, impondo-se a extração de seis fatores e definindo-se o fator apenas pelos itens cujas cargas fossem iguais ou superiores a 0,20 . As cargas fatoriais da matriz rotada são apresentadas na Tabela 5.

Figura 1- Visualização dos eigenvalues dos fatores extraídos na análise fatorial.

Tabela 5. Matriz de cargas fatoriais entre as variáveis e os componentes extraídos.

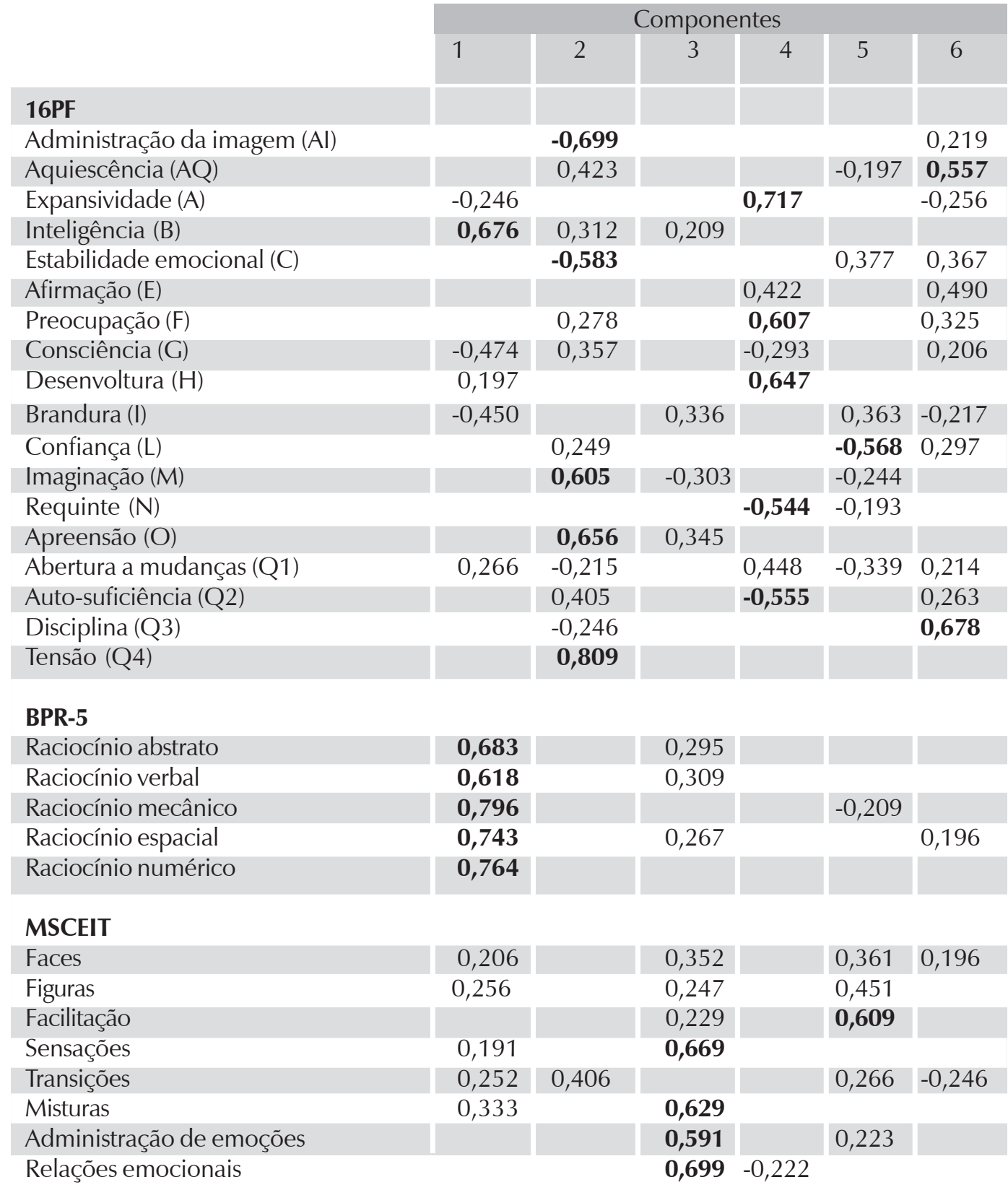

Nota. Em negrito, cargas maiores do que 0,50. 
Das quinze variáveis que apresentaram alguma carga no fator 1 , segundo os critérios definidos, seis se destacam por apresentarem cargas superiores a 0,6 e caracterizarem esse fator como relacionado, principalmente, às medidas de inteligência. São elas as cinco provas de raciocínio da BPR5 e o Fator B do 16PF. É interessante notar que, embora com magnitudes pequenas, existem alguns traços de personalidade que apresentaram carga nesse fator e parecem ser características associadas à observação do mundo de um ponto de vista lógico, racional, prático, centrado em objetos ao invés de pessoas. São as seguintes características (em ordem de magnitude das cargas): praticidade, objetividade e realismo (I-); abertura a novas experiências, tendência ao liberalismo $(\mathrm{Q} 1+)$; tendência à reserva, impessoalidade, distância e interesse por idéias e objetos ao invés de relações sociais (A-). Ao lado disso, cinco dos oito subtestes de inteligência emocional apresentaram cargas positivas e moderadas nesse fator, mostrando que essas medidas estão associadas à inteligência. A maior carga foi encontrada para o teste Misturas, que consiste em uma habilidade de compreensão emocional mais próxima da inteligência cristalizada (Primi, 2003).

O segundo fator agrega apenas traços de personalidade e, pela análise do conteúdo dos traços que se agrupam, pode-se entendê-lo como o traço geral comumente chamado de neuroticismo (fator global ansiedade no 16PF). As principais características agrupadas nesse fator são as seguintes (em ordem de magnitude das cargas): tensão, impulsividade e impaciência (Q4+); propensão à admissão de comportamentos e características indesejáveis (AI-); apreensão, indecisão, perturbação e propensão ao sentimento de culpa $(\mathrm{O}+)$; tendência à imaginação e atenção voltada para as idéias e associações internas ao invés dos estímulos externos $(\mathrm{M}+)$; baixa estabilidade emocional (C-) e baixo nível de consciência, tendência a comportamentos evasivos (G-).

O Fator 3 agrupou, principalmente, medidas de inteligência emocional com ênfase na área estratégica, uma vez que os subtestes com cargas fatoriais mais altas foram aqueles que envolvem habilidade de incorporar as emoções no processo de decisão para saber quais, dentre as ações alternativas, são mais adequadas para regular as emoções em si mesmo (administração de emoções) ou regular as emoções de outras pessoas (relações emocionais) e também a compreensão de emoções (transição e misturas). Uma exceção nesse grupo foi a tarefa sensação, da área experiencial, que apresentou carga fatorial alta nesse fator de gerenciamento emocional, mas também todos os outros subtestes do MSCEIT apresentaram cargas nesse fator, embora de menor magnitude.

É interessante notar que algumas medidas de personalidade e inteligência também apresentaram cargas acima de 0,20 nesse fator. Com relação aos traços de personalidade, esse fator associa-se às seguintes características (em ordem de magnitude das cargas): consciência, seguimento da moral convencional $(\mathrm{G}+)$; preocupação e propensão ao sentimento de culpa $(\mathrm{O}+)$, ao sentimentalismo, à compreensão empática baseada no sentimento $(I+)$; tendência a estar preso às sensações e percepções concretas externas e a guiar-se pela realidade objetiva e menos por abstrações (M-). 
Essas correlações sugerem algumas interpretações interessantes para significado desse fator. Segundo Cattell (1989), os fatores G e O compõem o construto freudiano superego, concebido como uma série de regras consensuais de valores culturais internalizadas que se conformam tanto como conteúdo quanto como ações. Os conteúdos se constituem por representações mentais de conhecimentos consensuais sobre valores endossados pela cultura (que, em última instância, formam a imagem de um "eu idealizado"). As ações são constituídas por mecanismos de restrição de impulsos egoístas que funcionam como agência de controle do comportamento, colocando-o subserviente ao dever (representado pelos conteúdos-regras) antes da gratificação pessoal (as experiências interiores dessas ações são nomeadas habitualmente por "voz da consciência").

O fator $\mathrm{G}$ indica o acordo com asserções da moral convencional e o fator $\mathrm{O}$ indica o sentimento de culpa em reação à desaprovação do superego. É importante frisar o conceito de "moral convencional", pois esse fator não indica a moral como princípios éticos universais como definido nos estágios mais avançados, segundo Kolberg, mas, sim, os estágios intermediários da moral convencional, indicando a aceitação, muitas vezes "irrefletida", das regras consensuais sobre valores culturais gerais, definidas, por exemplo, nos documentos sobre as leis sociais (Lukjanenko \& Primi, 2000).

É interessante notar as semelhanças entre os subtestes da inteligência emocional e os fatores de personalidade em termos de processos mentais subjacentes que tais medidas poderiam ter em comum. Ambas as medidas referem-se a mecanismos de regulação do comportamento e à aquiescência a um conjunto de conhecimentos socialmente consensuais sobre ações envolvendo experiências emocionais promotoras do bem-estar social; assim, as correlações têm um sentido teórico coerente com o que se espera para esse construto da inteligência emocional, corroborando a convergência encontrada desse fator com as medidas de personalidade.

Os dois outros traços de personalidade presentes no terceiro fator também indicam aspectos em comum com o construto medido. O fator I+ indica uma preferência para o processamento de informações envolvendo atenção aos elementos emocionais, sentimentais e subjetivos, características que facilitam a empatia e a compreensão dos estados de vulnerabilidade dos outros (Cattell \& Cattell, 1997). Tal processo coincide com a definição da faceta do gerenciamento emocional, como a abertura às experiências emocionais e a inclusão das informações provenientes dessas experiências nos processos de raciocínio, atingindo, com isso, tomadas de decisões mais eficazes (Mayer, Salovey \& Caruso, 2002). Também é interessante notar, como já foi falado, que o traço I do 16PF apresentou carga fatorial inversa no fator 1 , associado às medidas tradicionais de inteligência. Talvez isso seja coerente com a clássica contradição entre os dois tipos de inteligência: a tradicional, mais fria e objetiva, e a emocional, complacente à compreensão aos aspectos sentimentais e subjetivos. 
Pontuações baixas no traço M (16PF) indicam uma orientação perceptual aos eventos do mundo externo, uma orientação às informações práticas, objetivas da ordem do senso comum ao invés de preocupações com abstrações introspectivas. Tal associação sugere que esse fator da inteligência emocional associa-se mais à inteligência social do que ao conhecimento emocional interno, introspectivo, como definido pela inteligência intrapessoal de Gardner (1995), por exemplo. Esse fator também está associado, em menor magnitude, às medidas de inteligência (RV, RA, RE e Fator B), sendo que a associação mais forte foi com a prova de raciocínio verbal, que mede raciocínio com informações cristalizadas, o que é condizente com a faceta do conhecimento emocional.

O fator 4 foi denominado extroversão, já que somente medidas do 16PF tiveram cargas fatoriais iguais ou maiores que 0,2 e os subtestes principais são os que definem o fator de segunda ordem com o mesmo nome no 16PF (Russel \& Karol, 1999). Nomeadamente, os fatores foram: expansividade, tendência ao envolvimento emocional caloroso com os outros $(\mathrm{A}+)$; falta de timidez, busca por excitação, desenvoltura, insensibilidade às repreensões $(\mathrm{H}+)$; tendência à espontaneidade, orientação para a busca de prazer, tendência à despreocupação, alegria e animação $(\mathrm{F}+)$; tendência a buscar companhia e depender das opiniões dos outros para receber suporte, contato humano e direção, afiliação e sectarismo (Q2-); tendência à autenticidade, sinceridade e simplicidade (N-). Também foram agrupados, nesse fator, outros dois traços que não entram na composição do fator de segunda ordem extroversão, no
16PF, nomeadamente, a abertura às experiências, renovação e liberalidade $(\mathrm{Q} 1+)$ e afirmação, dominância, agressividade e assertividade $(E+)$.

A composição desse fator isolado das outras medidas é um resultado interessante porque distingue as medidas de inteligência emocional do construto extroversão. De fato, existe apenas uma correlação pequena e negativa com o subteste relações emocionais do MSCEIT. Isso pode sugerir que as interações sociais ligadas à extroversão são, antes de tudo, meios instrumentais de satisfação pessoal, o que, não necessariamente, culminará em atitudes compromissadas com uma compreensão empática do outro. Além disso, esse resultado é diferente do que geralmente se encontra nos estudos que empregam medidas de inteligência emocional por autorelato, que freqüentemente encontram correlações positivas com medidas de extroversão (Saklofske, Austin \& Minski, 2003).

No fator 5, observou-se uma composição mista de medidas de inteligência emocional e de personalidade. Por parte da inteligência emocional, as provas de facilitação, figuras e faces apresentaram as cargas fatoriais mais elevadas. As provas de transição e gerenciamento também apresentaram cargas baixas, mas acima de 0,20. Esse fator, portanto, está mais associado à área experiencial da inteligência emocional, significando a eficácia com que as atividades cognitivas de solução de problemas são informadas pelas experiências emocionais e também à capacidade de decodificar sinais emocionais consensuais em faces e figuras. A respeito dos fatores de personalidade, esse 
fator associou-se principalmente à tendência a ser confiante e tolerante com os outros e a acreditar nas características positivas (L-). Observou-se, também, uma associação menor com a força do ego, estabilidade emocional, capacidade de adaptação balanceada e tolerância ao estresse $(\mathrm{C}+)$; ao sentimentalismo, à compreensão empática baseada no sentimento $(\mathrm{I}+)$; ao convencionalismo, conservadorismo e pouca tolerância à mudança (Q1-).

A associação encontrada entre o fator $L$ e a faceta percepção das emoções sugere uma interpretação interessante dos processos subjacentes a essas medidas. O lado positivo do fator $\mathrm{L}$, que se associou negativamente a inteligência emocional, indica a existência de distúrbios nas relações interpessoais em razão de desconfiança generalizada das pessoas. Essa desconfiança tem origem em sentimentos mais profundos de privação, de comparação social desfavorável, insegurança e impotência para reconquistar aquilo que é percebido como objeto de privação, que dão origem a mecanismos de projeção defensiva que: "reconstroem a ameaça interna como um inimigo vindo de fora. Essa externalização requer que essas pessoas se tornem não somente hipervigilantes mas que elas também devam ser capazes de descobrir indícios asseguradores de que o perigo realmente existe ... a suspeição das pessoas que obtêm escores altos no fator $\mathrm{L}$ significa que elas são observadoras enviesadas, que ficam freqüentemente buscando evidências confirmatórias daquilo que pensam" (Cattell, 1989, p. 174).

Portanto, a suspeição não tem base na realidade, e, sim, em mecanismos intrapsíquicos defensivos, relativamente autônomos, que operam para regular a autoestima, mas à custa da percepção objetiva da realidade. A semelhança interessante com a inteligência emocional é que tais pessoas possuem deficiências perceptuais das relações sociais, o que é confirmado pela baixa habilidade na área experiencial, que envolve a percepção consensual de emoções em faces e imagens. Tais pessoas provavelmente têm uma percepção mais idiossincrática e diferente das concepções consensuais, que são tomadas como corretas nesses subtestes, porque, com freqüência, distorcem as percepções para que fiquem congruentes com o que precisam pensar para se sentirem bem, ainda que, em última instância, esse estado não indique bem-estar psíquico saudável.

Ainda, o lado positivo do fator $L$, em conjunto com o fator $\mathrm{C}$, são indicadores de capacidade de adaptação e bem-estar psíquico (Cattell, 1989; Cattell, Eber \& Tatsuoka, 1970), e fazem parte do fator neurose (ansiedade no 16PF) no sentido positivo (ausência de ansiedade). O fator I também apresentou carga positiva nesse fator, assim como no fator 3 , indicando a integração das emoções no processo de raciocínio como detalhado anteriormente. Ao lado disso, a prova de raciocínio mecânico da BPR-5 apresentou carga fatorial negativa e baixa nesse fator, o que, mais uma vez, indica que alguns aspectos constituintes do fator tradicional de inteligência têm uma tendência a apresentar uma relação inversa com os fatores da inteligência emocional. É interessante notar que essas características (Prova RM e fator I) estão associadas ao gênero, assim como a inteligência emocional (Bueno et al., em preparação). 
O sexto fator apresentou um conglomerado de variáveis cujo sentido não apareceu claramente interpretável, mas que parece indicar um aspecto da personalidade. As cargas mais altas nesse fator foram de autocontrole $(\mathrm{Q} 3+)$, aquiescência $(\mathrm{AQ}+)$ e afirmação $(E+)$. Uma possível interpretação refere-se a uma tendência mais ativoagressiva de orientação para realizações, preocupação com imagem pública $(\mathrm{Q} 3+)$, dominância e desejo de exercer papéis de controle $(E+)$, espontaneidade e busca por prazer $(\mathrm{F}+)$.

Em resumo, os resultados apresentados são semelhantes aos encontrados em estudos anteriores, também avaliando inteligência emocional por desempenho, nos quais foram encontrados dados indicando correlações, de moderadas a mínimas, medidas tradicionais de inteligência, configurando um padrão condizente com uma habilidade específica (Mayer, Caruso \& Salovey, 2000; Maccann et al., 2004; Jesus Jr., 2004; Côbero, 2004), e também demonstrando que, apesar de se encontrar uma certa covariância com traços de personalidade, não há indícios de que esses dois construtos se sobreponham (Roberts, Zeidner \& Matthews, 2001; Maccann et al., 2004; Jesus, Jr., 2004, Dantas, 2004; Côbero, 2004).

\section{Considerações finais}

O objetivo do estudo foi investigar a validade convergente-discriminante do MSCEIT com medidas de personalidade e inteligência. Pôde-se verificar, por meio de uma análise fatorial, que a inteligência emocional foi representada por dois fatores ortogonais (fator 3 e fator 5) aos outros quatro, ligados às medidas de inteligência (fator 1) e às medidas de personalidade (duas das quais, os fatores 2 e 4, bem conhecidos na literatura) o que sustenta a idéia de um construto diferente das medidas tradicionais de inteligência e personalidade usadas aqui.

Também foram encontradas algumas cargas fatoriais significativas de testes de inteligência e de personalidade nos fatores de inteligência emocional. Esses dados apontam uma convergência mínima que sustenta o pressuposto que inteligência emocional possa ser considerada um construto distinto referindo-se a uma capacidade específica. As correlações, especialmente com traços de personalidade, sugeriram interpretações sobre quais processos da personalidade estão em operação no ato emocionalmente inteligente, e foram condizentes com as concepções teóricas para os dois construtos isolados, um ligado à regulação emocional e outro à percepção das emoções, consistindo em evidência positiva de validade de construto.

A atual situação contraditória de testes de inteligência emocional, baseados em desempenho, que apresentaram baixas correlações com traços de personalidade, e testes auto-relato, que apresentaram correlações mais altas, pode indicar que o construto de inteligência emocional trate de aspectos do funcionamento mental que integra processos cognitivos e emocionais, e, por isso, situa-se a meio caminho entre esses dois grandes aspectos do funcionamento mental. Assim, os testes de auto-relato tratam de construtos da personalidade que prevêem adaptação inteligente ao meio, e os testes de desempenho tratam de construtos mais próximos de processos cognitivos 
envolvendo elementos emocionais. ̇̀ necessário, no entanto, um esforço mais aprofundado de entendimento dessas relações, já que os resultados encontrados neste estudo mostram que as associações entre inteligência emocional e os fatores da personalidade, quando melhor compreendidas, clarificam a interpretação dos processos subjacentes desse construto.

Os resultados encontrados neste estudo permitem um posicionamento mais favorável em relação à existência do construto inteligência emocional que, ao longo desses quinze anos de sua criação, tem sido muito contestado e desacreditado por vários pesquisadores, que realmente encontraram, em seus trabalhos, indícios que colocaram em dúvida a sua existência. No entanto, após o desenvolvimento dos instrumentos que avaliam inteligência emocional por desempenho, considerando-a uma habilidade, os resultados mostram-se mais promissores para essa área.

\section{Ricardo Primi}

Programa de Pós-graduação Stricto Sensu em Psicologia, Universidade São Francisco

As atividades de pesquisa deste autor que deram origem a este artigo são financiadas pelo CNPq e pela FAPESP.

José Maurício Haas Bueno

Universidade Presbiteriana Mackenzie e Universidade São Francisco

Monalisa Muniz

Doutoranda em Avaliação Psicológica, Universidade São Francisco, bolsista pela FAPESP

Universidade São Francisco, Laboratório de Avaliação Psicológica e Educacional (LabAPE), Mestrado em Psicologia, Rua Alexandre Rodrigues Barbosa, 45, CEP 13251-900, Itatiba, São Paulo, Fone (OXX11) 45348118,

E-mail: ricardo.primi@saofrancisco.edu.brou rprimi@uol.com.br. 
ALMEIDA, L. S. O Raciocínio: Delimitação do Conceito e sua Avaliação. In Almeida, Leandro S. O Raciocínio Diferencial dos Jovens. Porto: Instituto Nacional de Investigação Científica, 1998.

AUSTIN, E.J.; SAKLOFSKE, D.H.; HUANG, S.H.S.; MCKENNEY, D. Measurement of Trait Emotional Intelligence: Testing and Cross Validating a Modified Version of Schutte et al.'s (1998) Measure. Personality and Individual Differences, vol. 36(3), London: Elsevier, 2004, pp.555-562.

BARCHARD, K.A. Emotional and Social Intelligence: Examining its Place in the Nomological Network. Dissertation Abstracts International: Section B: the Sciences and Engineering, vol. 63(8 B), Yale: Yale University Library, 2003, p. 3950.

BARON, R. BarOn Emotional Quotient Inventory. Toronto: MultiHealth Systems, 1996.

BARON, R. Development of the Bar-On EQ-i: a Measure of Emotional and Social Intelligence. Artigo apresentado na $105^{\text {th }}$ Annual Convention of the American Psychological Association, Chicago, 1997

BRACKETT, M.A. \& MAYER, J.D. Convergent, Discriminant, and Incremental Validity of Competing Measures of Emotional Intelligence. Personality and Social Psychology Bulletin, vol. 29, no.9, 2003, pp.1147-1158.

BUENO, J.M.H. \& PRIMI, R. Inteligência Emocional: um Estudo de Validade sobre a Capacidade de Perceber Emoções. Psicologia: Reflexão e Crítica, 16 (2), 2003.

BUENO, J.M.H.; SANTANA, P.R.; ZERBINI, J. \& RAMALHO, T.B. (em preparação). Inteligência Emocional em Universitários.

CATTELL, H. B. \& CATTELL, H. E. P. The 16PF Cattell Comprehensive Personality Interpretation Manual. Illinois: Institute for Personality and Ability Testing, 1997.

CATTELL, H. B. The 16PF: Personality in Depth. Illinois: Institute for Personality and Ability Testing, 1989.

CATTELL, R. B., CATTELL, A. K. S. \& CATTELL, H. E. P. Questionário 16PF. 5으. ed. Rio de Janeiro: CEPA, 1993.

CATTELL, R. B.; EBER, H. W. \& TATSUOKA, M. M. Handbook for the Sixteen Personality Factor Questionaire (16PF). Illinois: Institute for Personality and Ability Testing, 1970

CHAN, D.W. Dimensions of Emotional Intelligence and Their Relationships with Social Coping Among Gifted Adolescents in Hong Kong. Journal of Youth and Adolescence, vol. 32(6), 2003, pp.409 418.

CHICO, E. Evaluación Psicometrica de una Escala de Inteligencia Emocional. / Psychometric Evaluation of an Emotional Intelligence Scale. Boletín de Psicología Spain, vol. 62, mar.1999, pp.65 78.

COBÊRO, C. Inteligência Emocional: Validade do MSCEIT no Contexto Organizacional. Dissertação de mestrado. Itatiba, Universidade São Francisco, 2004.

DANTAS, M.A. Evidências de Validade do Mayer Salovey Caruso Emotional Intelligence Test (MSCEIT). Dissertação de Mestrado, Universidade São Francisco, Itatiba, 2004.

DAVIES, M.; STANKOV, L. \& ROBERTS, R.D. Emotional Intelligence: in Search of an Elusive Construct. Journal of Personality and Socia Psychology, 75(4), 1998, pp. 989-1015.

GARDNER, H. Inteligências Múltiplas: a Teoria na Prática. Porto Alegre: Artes Médicas, 1995.

JESUS JÚNIOR, A. G. Estudo de Validade e Precisão do Mayer Salovey - Caruso Emotional Intelligence Test. Dissertação de Mestrado, Programa de Pós-Graduação Stricto Sensu, Universidade São Francisco, Itatiba, 2004.

$\mathrm{KOHAN}, \mathrm{A}$. Emotional Intelligence: an Investigation of Discriminant and Concurrent Validity. Dissertation Abstracts International: Section B: The Sciences and Engineering, vol. 63(8-B), 2003, pp.3923.

LUKJANENKO, M. F. S. P. \& PRIMI, R. Estudo Piloto de um Instrumento de Avaliação do Juízo Moral entre Universitários. Psicologia Escolar e Educacional, 3 (3), 2000, pp.217-226.

MACCANN, C.; ROBERTS, R.D. .; MATTHEWS, G.; ZEIDNER, M. Consensus Scoring and Empirical Option Weighting of Performance Based Emotional Intelligence (EI) Tests. Personality and Individual Differences, vol. 36(3), 2004, pp.645 662

MAYER, J. D. \& SALOVEY, P. What is Emotional Intelligence? In P. Salovey \& D. J. Sluyter (eds.). Emotional Development and Emotional Intelligence: Implications for Educators. New York: Basic Books, 1997, pp.3-31.

MAYER, J. D., SALOVEY, P., \& CARUSO, D. R. Mayer-Salovey-Caruso Emotional Intelligence Test (MSCEIT) User's Manual . Toronto, Canada: MHS Publishers, 2002

MAYER, J. D.; CARUSO, D. \& SALOVEY, P. Emotional Intelligence Meets Traditional Standards for an Intelligence. Intelligence, 27(4), 2000, pp.267-298.

MAYER, J. D.; DIPAOLO, M.T. \& SALOVEY, P. PerceivingAffective Content in Ambiguous Visual Stimuli: a Component of Emotional Intelligence. Journal of Personality Assessment, 54, 1990, pp.772-781.

MAYER, J. D.; SALOVEY, P. \& CARUSO, D. Emotional IQ Test (CD ROM). Needham: MA, Virtual Knowledge, 1997.

MAYER, J.D.; SALOVEY, P.; CARUSO, D.R.; SITARENIOS, G. Measuring Emotional Intelligence with the MSCEIT V2.0. Emotion, vol. 3(1), 2003, pp. 97-105

PALMER, B.R.; MANOCHA, R.; GIGNAC, G.; STOUGH, C. Examining the Factor Structure of the Bar-On Emotional Quotient Inventory with an Australian General Population Sample. Personality and Individual Differences, vol. 35(5), 2003, pp.1191-1210.

PASQUALI, L. Psicometria: Teoria dos Testes na Psicologia e na Educação. Petrópolis, RJ: Vozes, 2003.

PETRIDES, K V; FURNHAM, A. On the Dimensional Structure of Emotional Intelligence. Personality and Individual Differences, vol. 29(2), 2001, pp. 313-320.

Trait Emotional Intelligence: Psychometric Investigation with Reference to Established Trait Taxonomies. European Journal of Personality, vol. 15(6), 2001, pp.425-448.

PRIMI, R. Inteligência: Avanços nos Modelos Teóricos e nos Instrumentos de Medida. Avaliação Psicológica, 2 (1), 2003, pp.67-77.

ROBERTS, R.D..; ZEIDNER, M.; MATTHEWS, G. Does Emotional Intelligence Meet Traditional Standards for an Intelligence? Some New Data and Conclusions. Emotion, vol. 1(3), 2001, pp. 196231.

RUSSEL, M., \& KAROL, D. 16PF-Quinta Edição-Manual do Examinador. Rio de Janeiro: CEPA, 1999.

SAKLOFSKE, D.H; AUSTIN, E.J.; MINSKI, P.S. Factor Structure and Validity of a Trait Emotional Intelligence Measure. Personality and Individual Differences, vol. 34(4), 2003, pp. 707-721.

SALOVEY, P. \& MAYER, J. D. Emotional Intelligence. Imagination, Cognition and Personality, 9, 1990, pp.185-211.

SCHUTTE, N.S.; MALOUFF, J.M.; HALL, L.E.; HAGGERTY, D.J. COOPER, J.T.; GOLDEN, C.J.; DORNHEIM, L. Development and Validation of a Measure of Emotional Intelligence. Personality and Individual Differences, vol. 25(2). 1998, pp.167-177.

SUTARSO, P. Gender Differences on the Emotional Intelligence Scale (EQI) (marital status). Dissertation Abstracts International: Section B: the Sciences and Engineering, vol. 60 (6B).1999, p.3011.

TAPIA, M. Measuring Emotional Intelligence. Psychological Reports, vol. 88(2). 2001, pp.353-364. 